\title{
Blue Monitor : A PEEM-based Online Battery Monitoring and Maintenance Platform
}

\author{
Chao Ren, Kaige Chang, Caihong Li(Corresponding Author) \\ School of Mathematics and Statistics, \\ School of Information Science and Engineering, \\ Lanzhou University \\ Lanzhou, 730000, China \\ rench08@lzu.edu.cn
}

\author{
Jing Bao \\ Lanzhou Branch, \\ Gansu Mobile, \\ China Mobile Limited \\ Lanzhou, 730000, China \\ 13919044444@139.com
}

\begin{abstract}
-with rapid development of the mobile telecommunications industry, numerous Power Equipment and Environment Monitoring System (PEEM) related products have emerged in recent years. These products provide convenient remote maintenance. However, most of the existing systems remain in the phase of collecting data, which is not very useful for further analysis to improve maintenance operations. In this paper, we introduce a PEEM-based online battery monitoring and maintenance platform called Blue Monitor system. By maintaining communication power supply system in the mobile base station, Blue Monitor can reduce fossil-fuel power generator usages, decrease operational difficulty, enable unattended monitoring service and extend battery life. It will significantly boost energy savings and financial benefits.
\end{abstract}

Keywords-Battery Maintenance; Battery Monitoring; Unattended Monitor; Online Maintenance;

\section{INTRODUCTION}

With the popularity of mobile phones and electronic products, mobile communication plays an important role in people's daily lives. It is well known that all mobile users access mobile network through base stations, which bridge all mobile users in a cell and connect mobile calls to the mobile switching center. In order to achieve station remote monitoring, many companies have developed their own products: PSMS of Emerson Company [1], PSMS of HUAWEI Company [2], CSC system of GALAXY ELECTRONIC Company [3], PSMS of GOSUN Company [4] and others [5] which focus on automated data collection system. "Today, numerous streams of data are being collected from sensors that monitor the environment. When used in conjunction with computational models, these streams can be important sources of data for understanding physical phenomena and human behavior [6]." All the existing systems act as a sensor network which consists of a variety of sensors, gathering battery signal, door hitting information, parameter of switched power and environmental message. The information collected will be transmitted via the Ethernet to enable remote monitoring. A common goal of these systems is to help maintainers viewing station status remotely while avoiding the need for someone to attend a room or the difficulty when the station power failure occurs.
The current situation is that the maintainer sits at the PC to observe the power supply of a station remotely. Once the power failure occurs, the maintainer give a call to commission maintenance for oil-generating; the commission maintenance will ask the status of the station, and only if he thinks that oil-generating may be prerequisite, he will go for oil-generating. However, this pattern is likely to result in significant waste of energy.

Energy problem is an important task in various fields since "the outline of national economic and social development for the eleventh five-year plan". [7] puts forward the goal of energy saving and emission reduction, every profession generalizes the corresponding program, the systems mentioned above achieves the goal in a large extent, but there are some problems as follows: 1) they cannot achieve complete unattended, because a lot of human resources also need to wait at the PC; 2) The switching power supply manufacturer doesn't understand the using environment of battery, consequently, the initial set is unified, but the initial set is often not changed in use that battery isn't in the best state and its service life is affected; 3 ) when the electricity of station goes off, station is supplied from the battery in it, commission maintenance may be afraid of the station power off, he will go to generate electricity for the station that might not be needed; 4) Communications group can only get the oil-generating data from maintenance agency company, and the data can't be integrated into PSMS, while leads to questionable credibility.

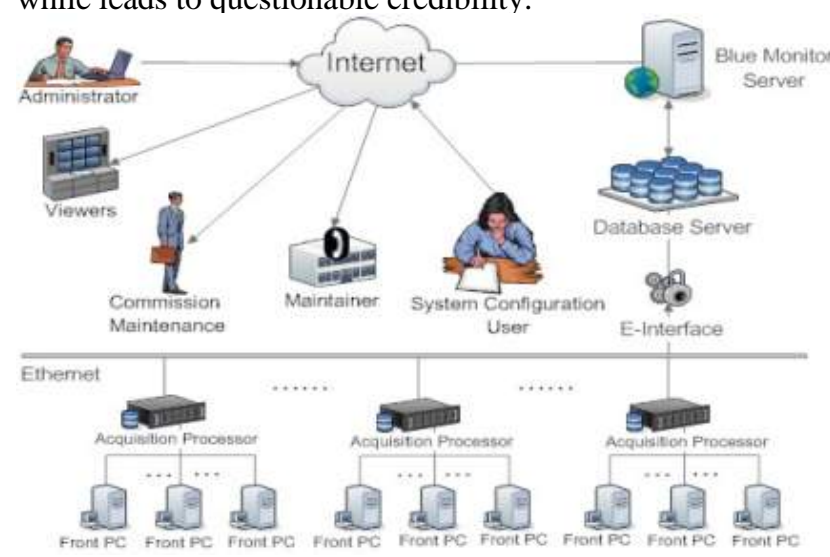

Figure 1. The Blue Monitor System Overview. 
Working with China Mobile Communication Corporation Lanzhou branch to address above problems, we propose the Blue Monitor system. Based on the combination of B / S structure and the C / S structure, the system uses Web 2.0 technology to improve user experience, and employs the $\mathrm{C} / \mathrm{S}$ structure to accomplish E-Interface and judge faults.

The rest of this paper is organized as follows: after providing an overview of the Blue Monitor system in Section II, we discuss the information process of the Blue Monitor system in Section III. In Section IV, we present a system demonstration. Finally, Section V concludes our paper and provides some future research directions.

\section{OVERVIEW OF THE BLUE MONITOR SYSTEM}

The physical architecture of Blue Monitor system is shown in Fig. 1. The data which collected by front PC transports into acquisition processors and Blue Monitor gathers data from there through E-Interface. After the process of the data processing, information is presented to different types of users. To ensure the system extensibility, we apply the highly-modular design to our system using the classic three-tier architecture: Presentation Tier, Application Tier, and Data Tier.

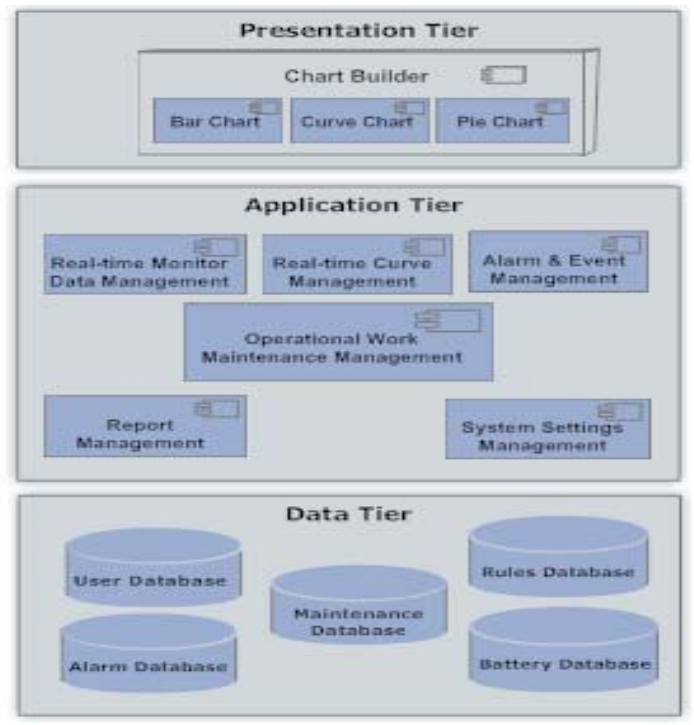

Figure 2. The UML Package Diagram of Information Flow.

\section{A. Presentation Tier}

First, confirm that you have the correct template for your paper size. This template has been tailored for output on the US-letter paper size. If you are using A4-sized paper, please close this template and download the file for A4 paper format called "CPS_A4_format". In presentation tier, we use Web 2.0 technologies including AJAX to develop a highly interactive Web User Interface for intuitively accessing the desired information. EXT JS [8] is used to fulfill the User Interface Style and Layout. Using Google DITU [9] as the mapping agent, we can easily embed maps to present station position including real-time battery information on the map. As shown in Fig. 2, our system provides several types of charts including curve chart, bar chart and pie chart to show Statistics Reports, this work can be easily done by Open Flash Chart [10].

\section{B. Application Tier}

We use IIS as our web application server, and ASP.NET as the main technology. For non-web application, C\# is used to implement the $\mathrm{C} / \mathrm{S}$ architecture. In order to add different modules to this tier according to specific user needs, we keep our architecture open. Currently, there are six default modules as shown in Fig. 2: Real-time Monitor Data Management (RMDM), Real-time Curve Management (RCM), Alarm \& Event Management (AEM), Operational Work Maintenance Management (OWMM), Report Management (RM) and System Settings Management (SSM).

- RMDM consists of three sub-modules: Station Management enables users to view real-time data of each station; E-Map helps users to intuitively observe the battery information of every station on the map. Equipment Management allows users to understand the equipment information including factory, model, parameters, and so on.

- RCM provides three main functions: user can examine the real-time data acquisition curve including voltage, electric current and capacity under different environmental conditions; user can also view the standard real-time data curve under different environmental conditions; oil-generating data curve under different environmental conditions can also been shown to user.

- AEM is composed of four sub-modules: Alarm Event Shield permits user to shield alarm event which is not relevant. Alarm Warning Management provides the functions of setting up alarm style, period and so on. Station Normal State-level Management indicates basic alarm information of each station. Station System State-level Management indicates important alarm information of each station, e.g. acquisition abnormal alarm, battery state of exception alert and oil machine scheduling alerts.

- OWMM is made up of six sub-modules: Expert Knowledge Base Maintenance allows exports submitting the maintenance advice into the system. Maintenance System Self-diagnosis is used to discover problems in the station and repair them automatically. Battery Chargers State Management controls the chargers methods for the battery so as to prolong the service life of a battery. Oil-generating Charge Manner Management avoids creating an issue for battery, because of wrong charge method. Station Normal State-level Maintenance puts forward the rational proposal for. Station Normal State-level Alarm the same as Station System Statelevel Maintenance.

- $\quad$ RM provides several types of statistical reports. User can easily find out failure statistics report, oilgenerating statistics report, distribution of power 
failure statistics report, system operation $\log$ and so on.

- $\quad$ There are nine sub-modules in SSM: Configuration Services, Configuration Tool, Power Management, E-Interface, Multi-database Rule Table, Data Replication Services, Data Backup / Recovery Services, Data Analysis Service and Configuration Upgrade.

\section{Data Tier}

SQL SERVER 2005 with built-in geospatial support is used as the principal database storage. In order to collect real-time battery data from different PSMS companies, we use Hibernate as the main technology of E-Interface. EInterface can transform the original data from different manufacturers into the same format. Different configuration is implemented by Hibernate using diverse XML files.

We currently manage conventional user profile information including username, user type, last access IP and time, telephone number, email address and privileges. The user profile information can be extended to improve the information verification capability in future.

Basic station information contains station name, latitude and longitude of station, equipment information, workload information, and urban district. For each station, the following information also needs to be recorded: electric current, voltage, AC state, three-phase voltage, acquisition time, environment temperature, average-charge voltage, float-charge voltage, and capacity.

Alarm information is essential in the Blue Monitor system. Alarm type, alarm time, alarm state, alarm rank, alarm confirm information, related person, related station information are also record in the alert table. For each type of alarm, we build the maintenance table to provide related person some rational proposal.

In order to determine battery capacity and obtain alarm type, we also need to build some rule tables. And these rules have to be verified by experiments and collected information. After that we record battery capacity under different conditions, such as electric current, voltage, environment temperature, and workload information. With continuous improvement, the rule table will become more useful, enabling Blue Monitor can determine the capacity of battery more accurately using the condition of power outage. Whether the battery is charging or not, if only the state is different from standard library, the system will give a certain alarm.

\section{INFORMATION PROCESSING}

In this section, we discuss how the Blue Monitor system collects and processes station battery information.

\section{A. Information Source}

There are two information sources in Blue Monitor system, battery maintenance experts and E-Interface.

- Battery Maintenance Experts: The experts of the Blue Monitor can submit maintenance suggestion into the system. If maintenance suggestion is sufficient, a knowledge-based expert system is built.
- E-Interface: Real-time battery information is collected from different PSMS systems by EInterface. Through E-Interface, data from different PSMS systems can be recognized as the same. We use the information gathered by E-Interface for further analysis.

\section{B. Informatin Inflow and Processing}

Fig. 3 is the UML activity diagram depicting the information inflow in the Blue Monitor system. First of all, it has to determine whether the data collected by E-Interface is charge data or not. If it is charge data, the system then pushes the data into the next stage to determine whether it meets the rules. If the data does not meet the rules or the data is not charge or discharge data, it will be pushed into alarm verification module. If it is an alarm, it will be recorded into the alarm table and the system will send out an alarm to the maintenance crew. Otherwise, the data is recorded into the rules table. If the data satisfies rules table, it will be pushed into fuel power generator schedule verification module. When the system recognizes that it needs electric generation, it will send a message to the maintenance crew; otherwise the system will record the data for the rules table. Above all, Fig. 3 illustrates a complete data collection process.

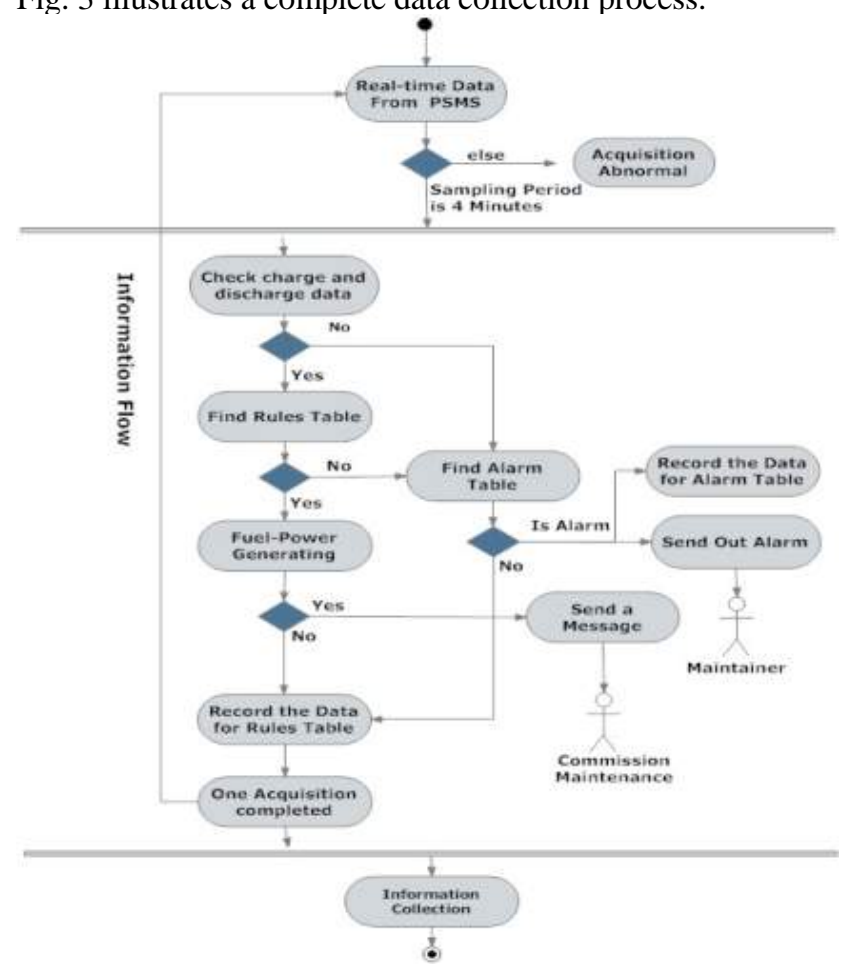

Figure 3. The UML Activity Diagram of Information Flow.

Compared with the existing systems, Blue Monitor not only collects the battery information, but also analyzes the collected data. It builds rules table and alarm table to decide whether the battery is damaged. Maintainer does not need wait at the PC, because it can send messages to him/her when something goes wrong. The system also can be monitored to determine if oil-generation is a prerequisite. If 
his endurance of a battery in the station is ok, Blue Monitor does not send the message to Maintenance Commission. In a sense, Blue Monitor is designed to cut down on wasteful costs.

\section{SYSTEM DEMONSTRATION}

As discussed in Section I, Blue Monitor combines the requirement of China Mobile Communication Corporation Lanzhou branch. The system connects 494 stations from the cities of Gannan, Linxia, Dingxi, and Baiyin. Due to the space limit, we only demonstrate part of the navigation of Blue Monitor as shown in Fig. 4. There are three important applications in Blue Monitor.

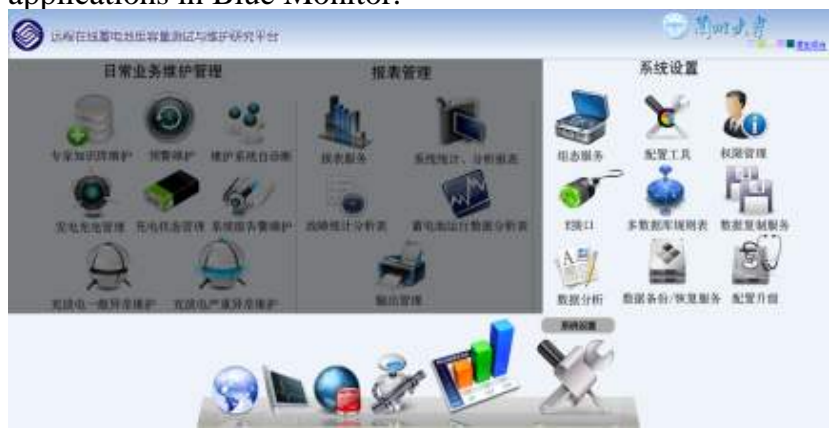

Figure 4. The Navigation of Blue Monitor.

\section{A. E-MAP}

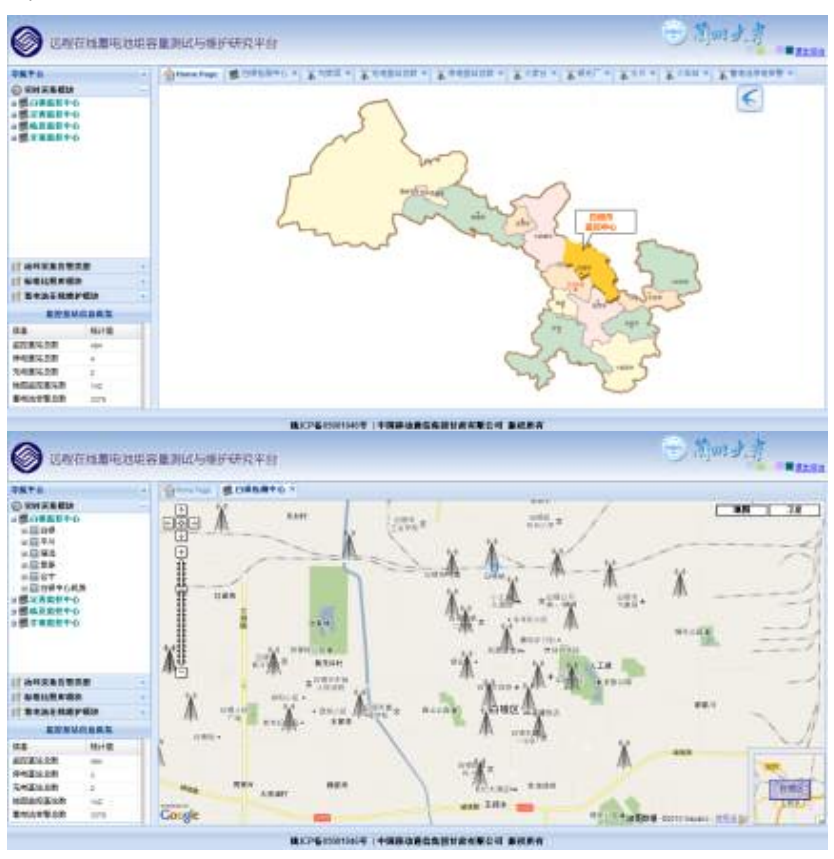

Figure 5. The E-MAP of Blue Monitor.

Geospatial information has been widely used in many fields. Using a provided map, we can easily find any desired place. As discussed in Section II, we use Google DITU in Blue Monitor to improve the user experience. The system controls 142 stations of Baiyin which contain latitude and longitude data. If there is something wrong with the station, it will be shown in red color in the map. The E-MAP UI is shown in Fig. 5. Using this map, maintainer can find the station she/he needs to pay attention quickly. If the error status always exists, the system will send message to related person until the error is fixed.

If the position of station is not right, only the authorized user can change the position by clicking on the right mouse button to select change the position information, and then drag the icon to the correct location. We can modify the station information in the same way, simply selecting the change station information menu.

\section{B. Alarm \& Event Management}

After conducting numerous practical charge/discharge and breach experiments, we have obtained a lot of useful data. We analyze the data and summarize the parameters from the data to build rules table and alarm table. Alarm information is used to remind the maintainer that the battery of this station is abnormal and indicates which kind of alarm is activated in the alarm message.

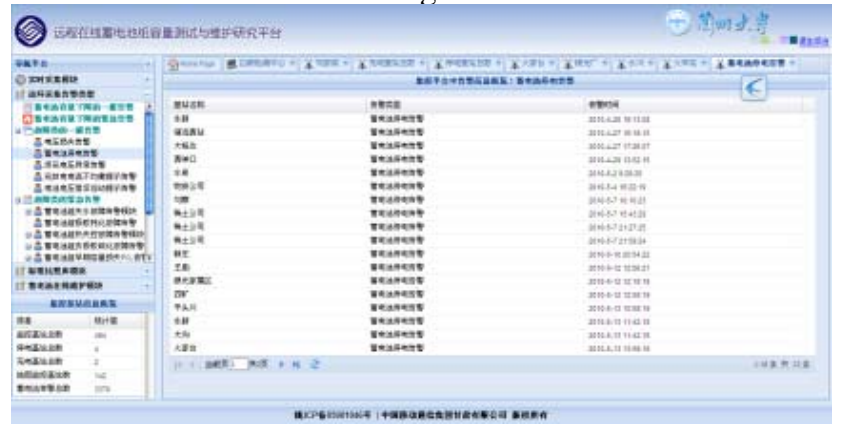

Figure 6. The Alarm of Blue Monitor.

The system summarizes history data and compares with rules table and alarm table to get the alarm information. As Fig. 6 shown, our system provides 24 types of alarms about battery. Among them 19 are general alarms in battery charge and discharge; 5 are serious alarms in battery charge and discharge. General alarm types contain: 1) battery voltage falls behind exceptionally low; 2) float-charge voltage anomalies; 3) float electric current over-current; 4) discharge electric current is not balanced; 5) voltage loss; 6) averagecharge voltage charge anomalies; 7) charging time exception; 8) float-charge voltage to average-charge voltage anomalies; 9) average-charge voltage to float-charge voltage anomalies; 10) frequently charging or discharging; 11) voltage of charging and discharging in high temperature; 12) voltage of charging and discharging in low temperature; 13) electric current of charging and discharging in high temperature; 14) electric current of charging and discharging in low temperature; 15) current / remaining capacity of charging and discharging in high temperature anomalies; 16) current capacity of charging and discharging in low temperature anomalies; 17) charging voltage of oil-generating anomalies; 18) charging power of oil-generating anomalies; 19) charging anomalies in oil-generating. Serious alarm types are made up of the following: 1) dehydration fault; 2) negative plate curing failure; 3) thermal runaway failure; 4) PCL early capacity loss; 5) plate passivation failure. 
When the alarm occurred, the system will deal with the alarm in one of two ways. If the alarm is normal and may not lead to losses, the system will record it in logs; the maintainer can view this log by logging onto the system. If the alarm is important and may cause losses, the system will send message to related person until the alarm disappeared.

\section{Station Management}

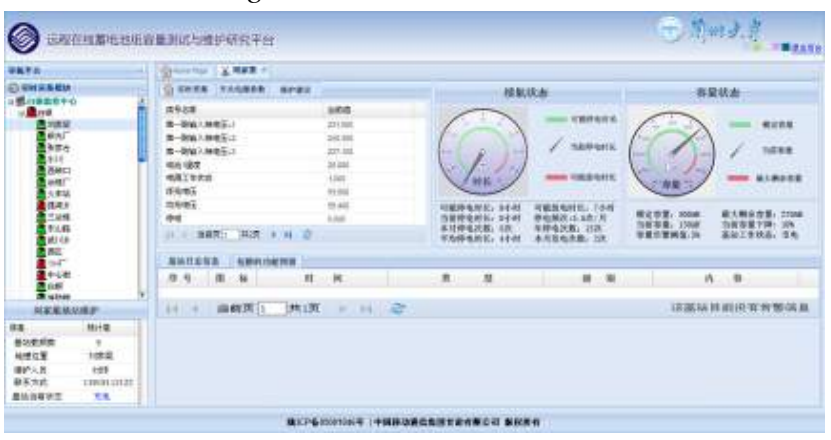

Figure 7. The Station Management of Blue Monitor.

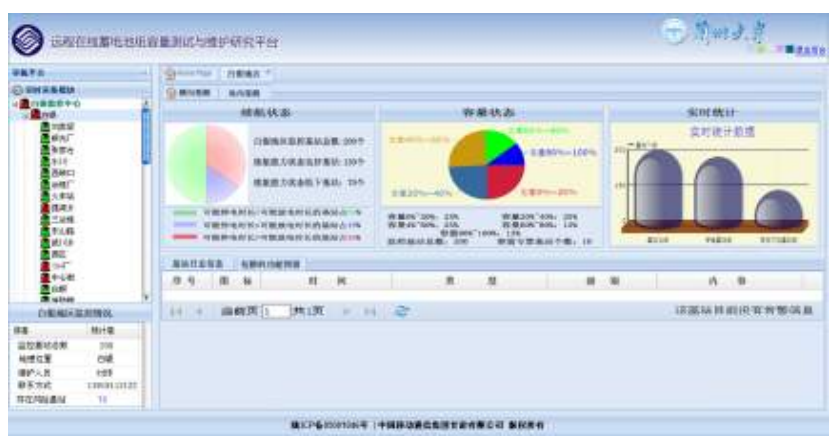

Figure 8. The Statistical Analyses of Station Management.

Station Management is a useful function for maintainer. As shown in Fig. 7, maintainer can use this function to monitor the real-time state of the battery for the station she/he is interested of, e.g. 'Liu Jialiang' Station. Furthermore, the maintainer can also check the duration and capacity status of this station. Duration status contains potential duration of blackout (PDB), potential duration of discharge (PDD), current duration of blackout (CDB), frequency of blackout, blackout times of this month, blackout times of this year. If PDB is greater than PDD, the battery in this station needs more care. Meanwhile, if PDB subtract CDB less than 2 hours, oil-generation is needed and the system will send a message to the Maintenance Commission through the SMS module. If PDB is less than
PDD, the battery of this station is safe, but needs to be checked. In other words, when CDB is greater than PDB, we need to pay more attention. Capacity status is made up of rated capacity, maximum spare capacity and current capacity. Capacity status is very important for duration status.

Blue Monitor's Station Management also provides statistical analyses based on information from the station. As displayed in Fig. 8, it shows the statistical information for city of Baiyin. All the station's physical condition contained in Baiyin region is showed visually in a user friendly format.

\section{CONCLUSIONS}

For the increasingly importance of energy savings in station management, this paper presents a system called Blue Monitor ,which is a PEEM-based online battery monitoring and maintenance platform. After providing a general overview of the entire system, this paper discusses the functions of this system in detail.

As this project is still in study phase, research work is still intensive. Currently many parameters for rules table and alarm table come from practical charge-discharge and breach experiments. We plan to pay more attention on the learning strategy research to make the system more robust.

\section{ACKNOWLEDGMENT}

This work was supported in part by the National Natural Science Foundation of China under Grant No. 90924025 and by the China Mobile Research Grant under Grant No. 2009_LH_05.

\section{REFERENCES}

[1] Emerson Web Site. http://www.emerson-ap.com/chi/home/index.asp

[2] HuaWei Web Site. http://www.huawei.com/cn/

[3] GALAXY ELECTRONIC Web Site. http://www.galaxy-elec.com/main/user/index.asp

[4] GOSUN Web Site. http://www.gosun.info/

[5] P Oksa, J Nummela, M Soini, L Sydänheimo, M., "Buidling-up an Automated Data Collection System”, UbiCC Journal, vol. 3 no. 3 2008.

[6] Chen-Ritzo, C-H, C. Harrison, J. Paraszczak, and F. Parr, "Instrumenting the Planet.", IBM Journal of Research \& Development, vol. 53, no. 3, Paper 1, 2009.

[7] Chinese Government News Web Site. http://www.gov.cn/gongbao/content/2006/content_268766.htm

[8] EXT JS. http://www.sencha.com/

[9] Google DITU API. http://code.google.com/intl/zh-CN/apis/maps/.

[10] Open Flash Chart. http://openflashchart.com/ 